Rapid Reviews COVID-19•

\title{
Review 2: "A year of \\ genomic surveillance \\ reveals how the SARS-CoV- \\ 2 pandemic unfolded in \\ Africa"
}

\section{Adriana Heguy 1}

${ }^{1}$ NYU Langone Medical Center: NYU Langone Health Pathology UNITED STATES

Published on: Aug 30, 2021

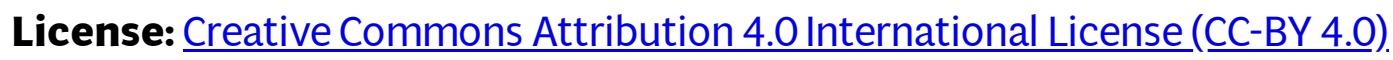




\section{$\underline{\text { RR:C19 Evidence Scale rating by reviewer: }}$}

- Strong. The main study claims are very well-justified by the data and analytic methods used. There is little room for doubt that the study produced has very similar results and conclusions as compared with the hypothetical ideal study. The study's main claims should be considered conclusive and actionable without reservation.

\section{$* * * * * * * * * * * * * * * * * * * * * * * * * * * * * * * * * * * * * * * * *$}

\section{Review:}

By $R R: C 19$ 's strength of evidence scale, the claims are reliable and supported by the data and methods used. Decision-makers should consider the claims in this study actionable with limitations based on the methods and data. This well-written manuscript represents an impressive genomic surveillance effort of African SARS-CoV2 sequences by a multinational set of researchers, labs, and centers spanning several continents. Even though it is understood that this represents a low sampling level given the true number of COVID-19 infections and the severity of the second wave of infections, the number of sequences analyzed in this study is close to 9000 (one in 300 officially reported cases) and this is a reasonably decent number to draw conclusions from regarding the variants of concern and variants of interest circulating in the continent and the dynamics of emergence and spread of these variants.

The phylogenetic and phylogeographic analyses are state-of-the-art in terms of the methodology used. The data shows a high number of early introductions from Europe in a manner similar to other places, like New York City, where the virus was also seeded multiple times-mainly from Europe. This early phase was followed by introductions from within African countries, with one of the major drivers being South Africa towards other African countries. The Beta variant, B.1.351, which was first detected in South Africa, is the variant present in the highest proportion as of May when this paper was uploaded to medRxiv. The manuscript emphasizes the importance of genomic surveillance to monitor the evolutionary dynamics of SARS-CoV-2 in the African continent, and this is an important endeavor that is highlighted right now by the rapid worldwide spread of the Delta variant. These efforts are important to determine future vaccination strategies and the composition of vaccines. The authors state that if the pandemic is not controlled in Africa, we may see the emergence of other variants that may escape at least part of the protection offered by vaccines-and this can affect not just Africa, but the entire world. 
